\title{
Características antropométricas, composición corporal y somatotipo en deportistas de élite de salvamento
}

\section{Anthropometric characteristics, body composition and somatotype in elite lifesaver}

\author{
J.Arturo Abraldes*, Nuria Rodríguez Suárez**, Carmen Ferragut Fiol*** y María Helena Vila Suárez ${ }^{* * * *}$ \\ *Universidad de Murcia, **Universidad Católica San Antonio, ***Universidad deAlcalá, ****Universidad de Vigo
}

\begin{abstract}
Resumen: El objetivo de este trabajo es triple: 1) Describir la estructura antropométrica, composición corporal y somatotipo de los mejores deportistas de salvamento. 2) Describir y comparar las características antropométricas de los deportistas de salvamento en función de sus especialidades, y 3) Comparar a los mejores deportistas de salvamento de Galicia con respecto a los especialistas de salvamento acuático del panorama nacional. La muestra empleada en este estudio fueron 85 deportistas de élite salvamento deportivo, de ellos 51 fueron hombres y 34 fueron mujeres. Los hombres presentaron una media de edad de $20.8 \pm 3.0$ años, $74.9 \pm 8.4 \mathrm{~kg}, 179.8 \pm 7.1 \mathrm{~cm}$ de altura y $185.2 \pm 9.8 \mathrm{~cm}$ de envergadura. Las mujeres presentaron una media de edad de $19.8 \pm 2.8$ años, $60.4 \pm 7.4 \mathrm{~kg}, 164.9 \pm 6.4 \mathrm{~cm}$ de altura y $166.4 \pm 9.9 \mathrm{~cm}$ de envergadura. Para el estudio se valoró el peso, la altura, la envergadura, cinco diámetros y cinco perímetros, calculándose el porcentaje graso, muscular y el somatotipo. Se aplicó un análisis de la varianza (ANOVA), con un post hoc de Tukey para analizar las diferencias entre categorías. Los deportistas de salvamento presentan un somatotipo mesomorfo balanceado en los varones y meso-endomórfico para las mujeres, siendo la mesomorfia el valor predominante en ambos casos. Existen diferencias entre la especialidad de playa y la de piscina. Los deportistas de selección gallega presentan un perfil antropométrico diferente al de los especialistas de piscina del panorama nacional.
\end{abstract}

Palabras clave: deporte, playa, piscina, natación, rendimiento, perfil deportivo.

\begin{abstract}
Abstrac: The aim of the study was threefold: 1) To describe the anthropometric profile, body composition and somatotype of the best sport lifesaving athletes 2) To describe and compare physical characteristics of the sport lifesaving athletes by discipline and 3) To compare the best Galician sport lifesaving athletes with the rest of Spanish sport lifesaving athletes. 85 elite sport lifesavers were recruited for the study, 51 men and 34 women. Physical characteristics of the sample were $20.8 \pm 3.0$ years; $74.9 \pm 8.4 \mathrm{~kg}$ and $179.8 \pm 7.1 \mathrm{~cm}$ height and $185.2 \pm 9.8 \mathrm{~cm}$ of arm span for male and $19.8 \pm 2.8$ years, $60.4 \pm 7.4 \mathrm{~kg}$ of weight, $164.9 \pm 6.4 \mathrm{~cm}$ of height and $166.4 \pm 9.9 \mathrm{~cm}$ of arm span for female. Additionally, 5 body girths and 5 skeletal breadths, somatotype, fat and muscle percentage were analysed. A one-way analysis of variance (ANOVA) and a Tukey post hoc test were used to determine if significant differences existed in the anthropometric profile among disciplines. Male sport lifesavers athletes show a balanced mesomorph and female sport lifesavers show a mesomorphic endomorph somatotype Male and female show predominance in mesomorph component. Our data suggest that there are differences between beach discipline and pool discipline. Galician sport lifesavers show differences in anthropometric profile with national pool specialist.
\end{abstract}

Key words: sport, beach, pool, swimming, performance, sport profile.

\section{Introducción}

Tradicionalmente, el propósito de la profesión de socorrista ha sido la de salvar vidas humanas. En la actualidad, el salvamento puede verse como una profesión y como un deporte, en el que se combinan elementos de la natación, remo, surf y carrera. Abraldes (2008) manifiesta que la actividad deportiva nace a raíz de la imitación de las técnicas utilizadas en rescates reales. Con el paso del tiempo se han ido desarrollado directrices, reglas y normativas, tanto para competiciones celebradas en aguas cerradas (piscinas) como en aguas abiertas (playas) existiendo, actualmente, un reglamento oficial auspiciado por la Federación Internacional de Salvamento (ILS). Este deporte, en España, va en aumento en la última década, celebrándose cada vez más competiciones deportivas y consiguiendo grandes éxitos a nivel internacional (Rodríguez, Abraldes \& Quintela, 2007). Sin embargo, esta evolución deportiva no va acorde con su producción científica, resaltando la necesidad de investigar en el rendimiento del salvamento deportivo. A día de hoy, apenas existen estudios científicos que profundicen en aspectos del rendimiento en esta modalidad deportiva.

Para conseguir el éxito en un deporte, se tiene que tener en cuenta multitud de factores que influyen (fisiológicos, psicológicos y biomecánicos, entre otros) en su rendimiento. Diferentes autores (Cabañas, 2009; Canda, 2012; Norton \& Olds, 2001; Vila et al., 2012) sugieren que cada deporte se define por unas características antropométricas particulares que posibilitan al-

Fecha recepción: 10-10-13- Fecha envío revisores: 13-10-13- Fecha de aceptación: 09-02-14 J. Arturo Abraldes

Calle Argentina, s/n

30720 - Santiago de la Ribera. Murcia.

abraldes@um.es canzar el alto rendimiento e incluso, dentro del mismo deporte, las diferentes especialidades tienen características físicas específicas (Bielec, Peczak-Graczyk \& Waade, 2013; Jürimäe et al., 2007; Leone, Lariviere \& Comtois, 2002). Son muchos los deportes que han sido estudiados e identificadas sus características físicas (Bayios, Bergeles, Apostolidis, Noutsos \& Koskolou, 2006; Gorski, Rosser, Hoppeler \& Vogt, 2014; Knechtle, Knechtle \& Rosemann. 2011; Liiv, Wyon, Jürimäe, Saar, Mäestu \& Jürimäe, 2013; Tsekouras et al., 2005). Aunque encontramos algunos estudios que han analizado las características fisiológicas de los deportistas de salvamento (Ferragut, Vila \& Rodríguez, 2007; Sinclair, Kerr, Spinks \& Leicht, 2009; Torras, Prats, Rodas, Riera, Viejo \& Alfaro, 1995), y antropométricas (Agrasar, Rodríguez \& Villar, 2003; Scanlan \& Dascombe, 2011; Vila, Rodríguez, Ferragut \& Abraldes, 2008), son escasos aquellos estudios que se centran en alguna especialidad concreta de este deporte (Fell \& Gaffney, 2001; Gulbin, Fell \& Gaffney, 1996) y que son específicas de un determinado país. Así es que, para definir el perfil del deportista de salvamento deportivo en general y de cada especialidad en particular, las referencias al rendimiento siempre se extraen de la comparación con otras modalidades afines al salvamento (Knechtle, Knechtle \& Rosemann, 2011; Martínez, Pasquarelli, Romaguera, Arasa, Tauler \& Aguiló, 2011; Morais et al., 2012; Rossi, Lyttle, El-Sallam, Benjanuvatra \& Blanksby, 2013). Esta ausencia de literatura específica, refuerza la necesidad de avanzar en el estudio de esta modalidad deportiva.

Antiguamente la competición deportiva buscaba al deportista más completo, que conseguía la mejor puntuación en la suma de las diferentes pruebas realizadas como un aspecto prioritario. Actualmente, la gran competencia, la multitud de pruebas y el aumento de clubes participantes, condiciona a un rendi- 
miento más específico por parte del deportista. Todo ello obliga a que, incluso los más jóvenes, comiencen a especializarse tempranamente en unas determinadas modalidades y pruebas (Abraldes, 2009). Los dos grandes bloques de pruebas deportivas se clasifican por el entorno de competición (piscina y playa), donde el dominio del medio acuático presenta características diferentes. Incluso, debido a la utilización de un material específico, deportistas de mayor edad, se especializan únicamente en algunas pruebas de una determinada modalidad deportiva. La mayoría de los estudios mencionados analizan el salvamento como un todo, sin embargo no profundizan en la especialización que comentamos. Siguiendo los argumentos de autores que aseguran diferencias incluso dentro del mismo deporte (Cabañas, 2009; Canda, 2012; Norton \& Olds, 2001; Vila et al., 2012) y ante la diversidad de pruebas en salvamento, se debe profundizar en el estudio del perfil deportivo de estas modalidades.

Galicia es una de las comunidades que más ha evolucionado en la última década en relación al número de deportistas, participación en competiciones y resultados (Rodríguez, Abraldes \& Quintela, 2007). De ahí que exista interés, en esta población, para analizar los parámetros que puedan indicar el rendimiento de los futuros deportistas, tratando de facilitar la detección del talento en edades tempranas. El análisis de la composición corporal es una forma fiable, práctica y no invasiva, para conocer aspectos que se modifican con el entrenamiento, y otros genéticos, no modificables por éste, que identifican las condiciones ideales del buen deportista (Canda, 2012; Esparza, 1993).

En base a los criterios descritos se plantearon tres objetivos fundamentales en esta investigación. El primero consistió en describir la estructura antropométrica, composición corporal y somatotipo de los mejores deportistas españoles en categoría sénior de salvamento deportivo. El segundo objetivo pretendió describir y comparar las características antropométricas de los deportistas en función de sus especialidades (piscina y playas). Finalmente, el tercer objetivo consistió en comparar a los mejores deportistas de salvamento deportivo de Galicia con respecto a los especialistas de salvamento acuático del panorama nacional. Todos los objetivos planteados en el estudio, se llevaron a cabo en función del sexo de los deportistas.

\section{Método}

\section{Muestra}

Un total de 85 deportistas de salvamento deportivo fueron analizados en este estudio. Todos ellos compitieron en el Campeonato de España de Salvamento deportivo de verano (Alicante, 2008), y pertenecen a la élite del salvamento deportivo. De los cuales 51 fueron varones con una edad media de 20.8 \pm 3.0 años, un peso de $74.9 \pm 8.4 \mathrm{~kg}$, una altura de $179.8 \pm 7.1 \mathrm{~cm}$ y $185.2 \pm 9.8 \mathrm{~cm}$ de envergadura; y 34 mujeres, con una edad media de $19.8 \pm 2.8$ años, un peso de $60.4 \pm 7.4 \mathrm{~kg}$, una altura de $164.9 \pm 6.4 \mathrm{~cm}$ y $166.4 \pm 9.9 \mathrm{~cm}$ de envergadura. La muestra se ha clasificado en cuatro grandes categorías: Especialistas en piscina (E. Piscina), Especialistas en playa (E. Playas), el resto de participantes del Campeonato de España (C. España) y la Selección Gallega (S. Gallega). En la Tabla 1 se puede observar las características demográficas en cada uno de los grupos citados y en relación al sexo.

Para determinar la selección de los grupos, tras la celebración del campeonato se ha revisado la puntuación de todas las pruebas y se ha otorgado una puntuación a cada uno de los participantes que han alcanzado los 8 primeros puestos en cada prueba. Sobre la puntuación obtenida, se han seleccionado a todos los que han obtenido una puntuación mayor a 25 puntos, siendo la máxima puntuación alcanzada 69 puntos para la playa y 60 en piscina, tan sólo por un deportista en cada especialidad.
El valor más repetido en los deportistas especialistas en playa ha sido 38 puntos, y de piscina han sido 41 y 54 puntos.

\section{Procedimiento}

Previamente a la toma de datos, todos los deportistas y el cuerpo técnico fueron informados de las pruebas que se iban a realizar, así como de los objetivos que se pretendían en la investigación. Todos los sujetos que participaron en la misma, firmaron un consentimiento informado en el que se les explicaba de las pruebas a las que se iban a someter y los objetivos específicos del trabajo. Los datos fueron registrados durante el Campeonato de Salvamento Deportivo de Verano en una sala anexa a la competición y acondicionada para el registro antropométrico (Esparza, 1993). Este estudio fue aprobado por el comité de ética de la Universidad Católica San Antonio de Murcia.

Las mediciones realizadas se ajustaron a los criterios, las normas, recomendaciones y técnicas de medida del International Working Group of Kinanthropometry (Stewart, Marfell-Jones, Olds, \& Ridden, 2011). Los evaluadores poseían la certificación de nivel I y II de la International Society for the Advancement of Kinanthropometry (ISAK). La evaluación de las características cineantropométricas se realizó en aquellos parámetros considerados importantes para el rendimiento deportivo en general (Malina, Bouchard, \& Bar-Or, 1991) y, para los deportes acuáticos en particular (Carter \& Ackland, 1994; Vila et al., 2008). Se analizaron principalmente dimensiones corporales como las longitudes (longitud del brazo y de la mano), perímetros (biacromial, biepicondíleo, biestiloideo, biiliocrestal, bicondíleo) las circunferencias (brazo relajado, brazo contraído, cintura, superior del muslo y medial del muslo) y los pliegues cutáneos (subescapular, tricipital, bicipital, pectoral, suprailíaco, abdominal, muslo y pierna medial.). Para la determinación del somatotipo se siguió el método antropométrico utilizado por Heath y Carter (1980).

El peso y altura se midió con una báscula y tallímetro SECA (SECA. Alemania) de precisión, con fracciones de 100g para el peso y de $0.1 \mathrm{~cm}$ para la talla. Los pliegues cutáneos se determinaron por triplicado mediante un plicómetro (Holtain Skinfold Caliper. Reino Unido), con 10 g.mm ${ }^{-2}$ de presión constante y un rango de $0.2 \mathrm{~mm}$. Se eligió como valor representativo de cada pliegue el valor medio de las tres mediciones. Aquellas medidas que se apartaron 2 ó más desviaciones estándar de la media fueron descartadas y nuevamente repetidas. Las lecturas del grosor de los pliegues se efectuaron hacia el $4^{\circ}$ segundo de la aplicación del plicómetro, para reducir la variabilidad asociada a diferencias de comprensibilidad cutánea. Los perímetros se registraron por triplicado (según protocolo de la ISAK), con una cinta métrica, inextensible, milimetrada y de fibra de vidrio (Lufkin Executive Thinline, W606PM, USA). Los diámetros (biestiloideo y bicondileo húmero, fémur y biacromial) se obtuvieron usando un antropómetro (GPM, Switzerland) con fraciones de $.01 \mathrm{~cm}$.

Para la determinación de la composición corporal se valoró el porcentaje graso a través de la fórmula de Carter (Carter, 1975), el peso muscular a través de la fórmula de Lee (Lee et al., 2000) y el índice de masa corporal (IMC) por medio del cociente peso/talla ${ }^{2}$, siendo el peso expresado en kilogramos $(\mathrm{kg})$ y la altura en centímetros (cm).

\section{Análisis estadístico}

Se analizó la distribución de la muestra en las distintas variables de estudio mediante el cálculo de estadísticos descriptivos básicos (media, desviación típica, valores extremos, etc.). Se realizaron tests de hipótesis (prueba de Kolgomorov-Smirnov y Lilliefors) y prueba de homogeneidad de Levene para todas las variables de estudio. También se llevó a cabo un análisis de la varianza multifactorial (Anova), con un post hoc de Tukey 
Tabla 1. Media y desviación típica corresp ondiente a las características antropométricas de deportistas masculinos de salvamento deportivo organizados por categorías. Diferencias signi ficativas: $\left.{ }^{*}\right)$ Respecto a los especial istas en piscina. $(\dagger)$ Respecto a los especialistas en playa

\begin{tabular}{|c|c|c|c|c|c|c|c|c|}
\hline \multirow[b]{3}{*}{ Características } & \multicolumn{4}{|c|}{ Mujeres } & \multicolumn{4}{|c|}{ Hombres } \\
\hline & E. Piscina & E. Playa & C. España & S. Gallega & E. Piscina & E. Playa & C. España & S. Gallega \\
\hline & $\mathrm{n}=8$ & $\mathrm{n}=4$ & $\mathrm{n}=11$ & $\mathrm{n}=11$ & $\mathrm{n}=11$ & $\mathrm{n}=8$ & $\mathrm{n}=26$ & $\mathrm{n}=6$ \\
\hline Edad (años) & $21.9 \pm 3.4$ & $19.8 \pm 2.6$ & $19.4 \pm 2.4$ & $18.7 \pm 2.3$ & $21.1 \pm 2.7$ & $22.2 \pm 4.3$ & $20.1 \pm 2.6$ & $18.5 \pm 2.3$ \\
\hline Peso $(\mathrm{kg})$ & $64.3 \pm 7.1$ & $56.9 \pm 3.4^{*}$ & $61.6 \pm 9.5$ & $58.8 \pm 5.1^{*}$ & $78.8 \pm 6.2$ & $67.1 \pm 7.1^{*}$ & $75.3 \pm 8.1$ & $72.5 \pm 14.5$ \\
\hline Altura (cm) & $169.7 \pm 7.3$ & $162.6 \pm 2.5$ & $165.2 \pm 8.1$ & $162.5 \pm 5.6$ & $182.2 \pm 5.2$ & $172.8 \pm 6.6^{*}$ & $180.7 \pm 6.8^{\dagger}$ & $177.5 \pm 7.1$ \\
\hline Envergadura (m) & $174.1 \pm 11.9$ & $164.7 \pm 5.1$ & $166.2 \pm 13.1$ & $162.4 \pm 7.5^{*}$ & $191.8 \pm 9.4$ & $177.6 \pm 9.4 *$ & $184.6 \pm 8.2$ & $179.2 \pm 6.6$ \\
\hline $\operatorname{IMC}(\mathrm{Kg} / \mathrm{m} 2)$ & $22.3 \pm 2.1$ & $21.5 \pm 1.2$ & $22.4 \pm 2.7$ & $22.3 \pm 1.7$ & $23.7 \pm 1.8$ & $22.5 \pm 2.1$ & $23.0 \pm 2.0$ & $22.9 \pm 3.6$ \\
\hline Carter (\%) & $18.5 \pm 2.3$ & $18.2 \pm 2.1$ & $18.7 \pm 5.2$ & $20.6 \pm 2.6$ & $9.6 \pm 1.6$ & $9.5 \pm 1.6$ & $10.6 \pm 3.1$ & $11.5 \pm 4.1$ \\
\hline $\begin{array}{l}\text { Lee (\%) } \\
\text { Longitudes }\end{array}$ & $24.3 \pm 2.8$ & $22.3 \pm 1.6$ & $23.9 \pm 4.5$ & $22.7 \pm 1.3$ & $34.8 \pm 2.1$ & $30.9 \pm 2.2^{*}$ & $32.5 \pm 2.2$ & $30.9 \pm 3.1^{*}$ \\
\hline Longitud brazo & $30.6 \pm 3.9$ & $30.0 \pm 1.6$ & $31.2 \pm 2.2$ & $30.6 \pm 1.7$ & $34.7 \pm 1.6$ & $31.8 \pm 2.5$ & $34.2 \pm 1.8$ & $33.9 \pm 1.5$ \\
\hline $\begin{array}{l}\text { Longitud mano } \\
\text { Perímetros }\end{array}$ & $18.7 \pm 1.2$ & $18.3 \pm 1.2$ & $18.0 \pm 1.6$ & $17.5 \pm 1.1$ & $20.1 \pm .8$ & $18.5 \pm .8$ & $19.0 \pm 1.9$ & $16.5 \pm 3.3^{*}$ \\
\hline Biacromial & $38.2 \pm 1.3$ & $36.2 \pm .3^{*}$ & $36.9 \pm 3.4$ & $37.0 \pm 2.3$ & $42.0 \pm 2.2$ & $40.5 \pm 2.6$ & $41.5 \pm 2.0$ & $39.6 \pm 1.7$ \\
\hline Biepicondíleo & $6.5 \pm .4$ & $6.2 \pm .2$ & $6.5 \pm .6$ & $6.2 \pm .3$ & $7.4 \pm .3$ & $7.0 \pm .2$ & $7.3 \pm .3$ & $7.1 \pm .3$ \\
\hline Biestiloideo & $4.8 \pm .6$ & $4.9 \pm .2$ & $5.0 \pm .5$ & $4.9 \pm .2$ & $5.8 \pm .2$ & $5.5 \pm .3$ & $5.7 \pm .3$ & $5.5 \pm .3$ \\
\hline Bi iliocrestal & $33.3 \pm 1.8$ & $31.4 \pm 1.0^{*}$ & $32.2 \pm 1.9$ & $33.1 \pm 1.3$ & $33.6 \pm 1.3$ & $32.4 \pm 1.3$ & $32.8 \pm 1.9$ & $31.1 \pm 4.0^{* t}$ \\
\hline $\begin{array}{l}\text { Bicondileo } \\
\text { Circunferenci as }\end{array}$ & $9.5 \pm .3$ & $9.1 \pm .6$ & $9.1 \pm 1.2$ & $9.1 \pm 1.1$ & $10.1 \pm .4$ & $9.6 \pm .6$ & $10.2 \pm .6$ & $10.1 \pm .9$ \\
\hline Brazo Relajado & $29.0 \pm 2.7$ & $27.1 \pm 1.4$ & $27.9 \pm 2.5$ & $28.0 \pm 1.6$ & $31.8 \pm 1.7$ & $30.8 \pm 2.7$ & $30.9 \pm 2.1$ & $29.7 \pm 3.4$ \\
\hline Brazo Contraído & $31.0 \pm 2.5$ & $29.1 \pm 1.3$ & $29.3 \pm 3.0$ & $28.9 \pm 1.4^{*}$ & $34.6 \pm 1.8$ & $33.1 \pm 1.7$ & $34.9 \pm 1.8$ & $32.5 \pm 2.5$ \\
\hline Cintura & $71.9 \pm 4.2$ & $69.9 \pm 2.6$ & $70.7 \pm 6.0$ & $70.1 \pm 3.3$ & $80.4 \pm 6.1$ & $76.7 \pm 4.6$ & $78.8 \pm 5.6$ & $78.5 \pm 8.6$ \\
\hline Superior Muslo & $58.3 \pm 3.5$ & $54.7 \pm 2.5$ & $58.1 \pm 4.9$ & $63.4 \pm 4.4^{* t}$ & $57.8 \pm 2.2$ & $54.4 \pm 3.5$ & $57.0 \pm 5.0$ & $56.3 \pm 9.4$ \\
\hline Medial Muslo & $50.6 \pm 2.5$ & $49.8 \pm 2.6$ & $52.0 \pm 4.0$ & $51.7 \pm 2.7$ & $53.4 \pm 1.9$ & $52.1 \pm 3.6$ & $52.9 \pm 3.4$ & $52.2 \pm 5.7$ \\
\hline So matotipo & & & & & & & & \\
\hline Endomorfia & $3.5 \pm .8$ & $3.2 \pm .4$ & $3.8 \pm 1.1$ & $4.3 \pm .5^{*+t}$ & $2.3 \pm .4$ & $2.7 \pm 1.3$ & $2.6 \pm 1.0$ & $3.1 \pm .5$ \\
\hline Mesomorfia & $4.7 \pm 1.1$ & $4.5 \pm .8$ & $4.3 \pm 1.0$ & $4.7 \pm 1.2$ & $5.1 \pm .9$ & $5.2 \pm .9$ & $5.0 \pm 1.2$ & $5.4 \pm 1.0$ \\
\hline Ectomorfia & $2.5 \pm 1.1$ & $2.4 \pm .6$ & $2.4 \pm .8$ & $2.0 \pm .9$ & $2.7 \pm .7$ & $2.6 \pm 1.1$ & $2.8 \pm 1.5$ & $2.9 \pm .9$ \\
\hline
\end{tabular}

muscular según Lee.

para analizar las diferencias estadísticamente significativas entre las distintas categorías de deportistas. Se consideró la existencia de diferencias estadísticamente significativas para una fiabilidad del 95\% ( $\mathrm{p} \leq .05)$.

\section{Resultados}

Entre los valores que caracterizan la muestra objeto de estudio (Tabla 1), se han encontrado diferencias estadísticamente significativas entre los especialistas de playa y piscina para las variables peso, altura y envergadura. Además, hemos hallado valores próximos a la significación en la envergadura, entre la selección gallega y los especialistas de piscinas.

Entre las deportistas femeninas se han encontrado diferencias estadísticamente significativas entre las especialistas de piscina con las especialistas de playa para el peso. Las deportistas de la selección gallega han presentado diferencias estadísticamente significativas con las especialistas de piscina para las variables peso y envergadura. También se han obtenido valores relevantes para la variable altura, sin llegar a la significación estadística.

Los varones especialistas en piscina presentan diferencias estadísticamente significativas en el peso muscular respecto a los especialistas de playa y a los seleccionados gallegos, que además difieren en la longitud de la mano. En las mujeres no hemos encontrado valores estadísticamente significativos, sin embargo, sí aproximación a éstos entre las deportistas gallegas y las especialistas de piscina, para las variables de porcentaje graso y longitud de mano. De mismo modo, no debemos des-

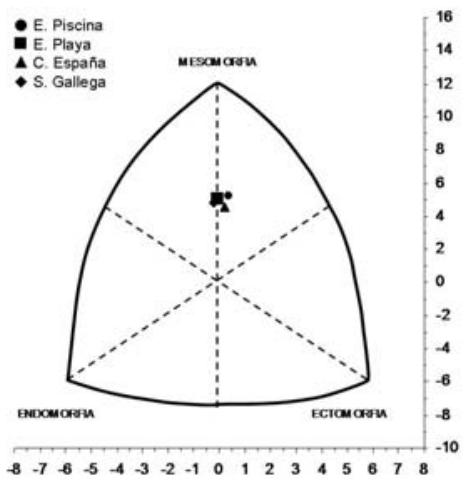

echar los datos entre especialistas de playa y especialistas de piscina para la variable longitud del brazo.

De los cinco perímetros analizados (Tabla 1), se han encontrado diferencias estadísticamente significativas entre varones de la selección gallega y los especialistas de playa y de piscina para la variable biiliocrestal. Los valores hallados del diámetro biepicondíleo entre varones especialistas en playa y piscina, también tiene fuerza sin llegar a la significación. Entre las mujeres deportistas se han encontrado diferencias estadísticamente significativas entre especialistas de playa y de piscina para las variables diámetro biacromial y biiliocrestal.

En relación a las circunferencias estudiadas no se han encontrado diferencias estadísticamente significativas para los grupos de deportistas varones. Solamente se han encontrado diferencias estadísticamente significativas en las mujeres, principalmente entre el grupo de deportistas gallegas y los especialistas en piscina (superior del muslo y brazo contraído) y playa (superior del muslo).

Tal y como se puede apreciar en la somatocarta (Figura 1), el somatotipo de todos los deportistas varones de salvamento deportivo fue el mesomorfo balanceado. Las mujeres deportistas presentaron un somatotipo meso-endomórfico para todos los grupos analizados, excepto para la selección gallega, que presentaron un somatotipo mesomorfo endomorfo (Figura 2). Se han encontrado diferencias estadísticamente significativas para la endomorfia entre las deportistas femeninas de la selección gallega y las especialistas de playa y piscina (Tabla 1).

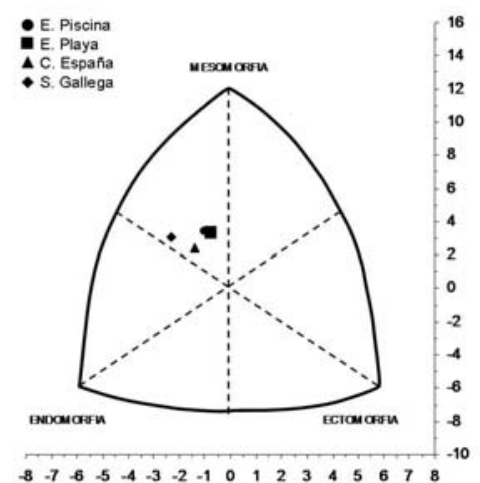

Figura 2. Kepresentacion aeı somatotipo ae ıs cuatro grupos analizaaos a e aeport stas mujeres.

\section{Discusión}

Tal y como se ha comentado en la introducción, son pocos los estudios científicos realizados a poblaciones específicas de salvamento deportivo. Además, la mayoría pertenecen a la década pasada y analizan características sin atender a su especialidad (Agrasar et al., 2003; Vila et al., 2008). Observamos en los resultados que los deportistas varones de salvamento deportivo presentan unas características antropométricas que sugieren la existencia de diferencias entre especialistas de playa y de piscina. Sin embargo, esta idea no se constata en los resultados hallados en las mujeres, que parecen ser más homogéneas en cuanto al tipo de competición en el que se desenvuelven. Por otro lado, las deportistas de la selección gallega presentan carac- 
terísticas diferentes con respecto a las especialistas de piscina. Generalmente los valores estadísticamente significativos son menores para las deportistas gallegas, lo que sugiere la existencia de variables demográficas (peso y envergadura, con importancia también de la altura) que expresan criterios para destacar en las pruebas de piscina. Así mismo, la existencia de valores diferentes en relación a las circunferencias (brazo contraído y superior del muslo). Mayores niveles de fuerza se relacionan con una mayor masa muscular, así como un mayor peso (González-Badillo \& Gorostiaga, 2002). Resultados opuestos a los encontrados en las mujeres gallegas permiten mayor nivel de fuerza en los brazos, adecuado para las técnicas de nado y, menor peso muscular, sobre todo a nivel de las piernas, facilitando la hidrodinámica en el nado y remolque (Chollet, 2003).

Los hombres especialistas en piscina presentan en todas las variables antropométricas estudiadas valores superiores que los especialistas de playa, encontrándose diferencias estadísticamente significativas para las variables que hacen referencia al peso, altura y envergadura. Valorando las pruebas que se realizan en la piscina los deportistas de salvamento deportivo, los resultados son similares a los presentados por nadadores, donde las longitudes son características importantes para el rendimiento (Bielec et al., 2013; Jürimäe et al., 2007; Morais et al., 2012; Rossi et al., 2013; Strzala \& Tyka, 2009). Estas características les permiten una mejor adaptación biomecánica, pues una mayor longitud de brazada es un factor decisivo en el rendimiento (Chollet, 2003; Silva et al., 2013).

También se han encontrado diferencias estadísticas en el peso muscular entre los varones especialistas de playa y los especialistas de piscina, lo que sugiere que la fuerza es una capacidad importante para el rendimiento. Estos resultados están en línea con lo expuesto por Scanlan y Dascombe (2011) y Morais et al., (2012) para las pruebas de aguas cerradas. La ausencia de diferencias estadísticas entre los diámetros analizados entre especialidades, y que todos los deportistas analizados compartan el mismo somatotipo, sugiere que las diferencias en el peso muscular pueden estar influenciadas a una mayor carga de entrenamiento por los especialistas de piscina. En este sentido, el nado en aguas abiertas está condicionado, no sólo a los niveles de fuerza sino también a las condiciones cambiantes del entorno (olas, corrientes, remolinos, etc.) que propician una mayor habilidad por parte del deportista especialista en pruebas de playa.

En el caso de los varones los resultados sugieren diferencias en ciertas características antropométricas entre las especialidades de piscina y playa. Estos resultados podrían estar fuertemente influenciados por la circunstancia de que en las pruebas de piscina muchos de los participantes son nadadores o exnadadores, mientras que para las pruebas de playa las necesidades y características para alcanzar el rendimiento no son las mismas (Abraldes, 2008; Ferragut, Vila \& Rodríguez, 2007). La ausencia de un mayor número de diferencias entre ambas modalidades, puede relacionarse con la falta de profesionalización de este deporte, lo que limita a los entrenadores y seleccionadores a la hora de realizar la selección de deportistas. Estas afirmaciones tienen una base empírica, y somos conscientes de que es necesario analizar con mayor profundidad las características de cada una de las pruebas. Resultados que ponen de relieve la necesidad de realizar un mayor número de estudios científicos sobre esta modalidad deportiva, en ambos sexos, para poder colaborar con los entrenadores, alcanzar el máximo rendimiento y evolucionar en el deporte.

En el caso de los deportistas masculinos pertenecientes a la selección gallega, presentan características antropométricas similares al resto de participantes de Campeonato de España, presentando diferencias estadísticamente significativas con los especialistas de piscina en dos variables no modificables por el entrenamiento (la envergadura y longitud de la mano). Como ya se ha mencionado anteriormente, son variables consideradas importantes para el rendimiento en natación (Martínez et al., 2011; Morais et al., 2012; Vila et al., 2008) y un referente a tener en cuenta para la detección del talento deportivo. Sin embargo, otra de las variables con diferencias estadísticamente significativas es en el peso muscular, ésta es una variable modificable por el entrenamiento, lo que sugiere la necesidad de intervención en el proceso de diseño de las cargas de entrenamiento (Scanlan \& Dascombe, 2011).

Las características antropométricas presentadas por las mujeres de salvamento deportivo no discriminan entre las especialidades de piscina con playa, aunque se sugiere que las longitudes podrían ser importantes para alcanzar el rendimiento en la modalidad de piscina, como ha ocurrido con los deportistas hombres. Esta interpretación debe hacerse atendiendo al contexto estudiado (cuatro deportistas especialistas en playas). Este bajo $n$ en deportistas mujeres atiende a la realidad del panorama español, donde practican menos mujeres que hombres salvamento deportivo a nivel de élite (Abraldes, 2009; Rodríguez, Abraldes \& Quintela, 2007).

Se confirma que el perfil de las mujeres de la selección gallega se diferencia del perfil especialistas en piscina. Estas últimas, presentan mayores longitudes (altura, envergadura, longitud de la mano), tienen menor porcentaje graso (Carter y endomorfia), y mayores valores en las circunferencias (circunferencia del brazo contraído y superior del muslo). Variables importantes en el rendimiento de la natación, donde los criterios de fuerza, flotación e hidrodinámica (Chollet, 2003) son muy representativos para obtener la mejor marca. Estos datos deben ser considerados por los entrenadores y cuerpo técnico, ya que son modificables con el entrenamiento y sus cargas. Del mismo modo, suponen una referencia para el seleccionador y para la detección de un futuro talento deportivo en salvamento acuático.

En un primer estudio (Torras et al., 1995), la selección nacional de salvamento acuático presentó valores inferiores en las variables de peso y altura para los hombres respecto a todas las categorías analizadas, excepto para los especialistas en playas. En el caso de las deportistas mujeres, los valores presentados por el estudio de Torras et al., (1995) son ligeramente superiores a los aquí reflejados, excepto para los hallados en las especialistas de piscina.

El somatotipo encontrado en los deportistas varones fue el mesomorfo balanceado en todas las categorías estudiadas. Este perfil no corresponde al presentado por otros estudios realizados en salvamento, donde el somatotipo del socorrista es el meso-ectomórfico (Torras et al., 1995) o endo-mesomórfico (Agrasar et al., 2003). Aspectos que, la mejora de los entrenamientos, y la evolución sufrida por éstos en este tiempo, hacen que el morfotipo se modifique en función de las nuevas necesidades que exigen los sistemas de trabajo y, en definitiva, este deporte. En el caso de las mujeres el somatotipo encontrado fue el meso-endomórfico para todas las categorías excepto para las seleccionadas gallegas, que presentaron un somatotipo mesomorfo-endomorfo. Otro argumento más que confirma la importancia que los niveles de fuerza presentan para el rendimiento deportivo, pues éste se encuentra representado en el somatotipo. En relación a la composición corporal, se ratifica la predominancia de la mesomorfía como característica de los deportistas, hombres y mujeres, de salvamento deportivo de alto nivel.

Somos conscientes de las limitaciones del estudio, centradas principalmente en la muestra y en sus categorías, pero la pretensión es aportar nuevos datos y un enfoque para seguir estudiando este deporte, tan escaso en publicaciones científicas, y conseguir la mejora y rendimiento del deportista en sus modalidades. 


\section{Conclusiones}

Los datos aportados en este trabajo sugieren que las mayores diferencias en este deporte hay que buscarlas no sólo en los parámetros analizados, sino en otros factores ajenos como la condición física, dominio técnico, etc. Por otro lado, no se consiguen establecer con certeza unos criterios de selección de deportistas al no poder contrastar y analizar muestras amplias de especialistas en este deporte. Sin embargo, podemos concluir dando respuestas a los objetivos planteados. En relación al primer objetivo se determinó la existencia de un somatotipo mesomorfo balanceado para los deportistas varones y somatotipo meso-endomórfico para las mujeres. El componente predominante en el somatotipo para toda la muestra de deportistas es la mesomorfia. Por otro lado, en base al segundo objetivo, se apreció que las características antropométricas establecen diferencias entre los mejores deportistas especialistas de piscina y de playas, principalmente en variables demográficas. Otras variables como el perímetro biacromial y biiliocrestal son importantes para diferenciar a ambos especialistas en deportistas mujeres. Finalmente, dando respuesta al tercer objetivo, se concluye que los deportistas de selección gallega presentan un perfil antropométrico diferente al de los y las especialistas de piscina del panorama nacional.

\section{Agradecimientos}

Queremos agradecer a todos los deportistas, seleccionadores y cuerpo técnico de las diferentes comunidades autónomas la colaboración prestada, sin su colaboración este estudio no habría podido realizarse.

\section{Referencias}

Abraldes, J.A. (2008). Salvamento Acuático y Deporte: Un estudio de los recursos humanos en las playas de Galicia, intervenciones en los rescates y su relación con el ámbito deportivo. La Coruña: Federación de Salvamento e Socorrismo de Galicia.

Abraldes, J.A. (2009). Formación deportiva en salvamento acuático. In vestigación en el Campeonato de España de autonomías. La Coruña: Federación de Salvamento e Socorrismo de Galicia.

Agrasar, C., Abraldes, J.A., Rodríguez, N., \& Villar, J.M. (2003). Estudio cineantropométrico de la composición corporal y el somatotipo de los componentes de la selección gallega de salvamento acuático deportivo. $3^{\text {er }}$ Congreso de Salvamento y Socorrismo de Galicia. Sanxenxo. Pontevedra.

Barlow, MJ., Findlay, M., Gresty, K., \& Cooke, C. (2014). Anthropometric variables and their relationship to performance and ability in male surfers. European Journal of Sport Science, 14(1), 177-177.

Bayios, I.A., Bergeles, N.K., Apostolidis, N.G., Noutsos, K.S., \& Koskolou, M.D. (2006). Anthropometric, body composition and somatotype differences of Greek elite female basketball, volleyball and handball players. Journal of Sports Medicine and Physical Fitness, 46(2), 271-280

Bielec, G., Peczak-Graczyk, A., \& Waade, B. (2013). Do swimming exercises induce anthropometric changes in adolescentes? Comprehensive Pediatric Nursing, 36, 37-47.

Canda, A. (2012). Variables antropométricas de la población deportista española. Madrid: Consejo Superior de Deportes y Ministerio de Educación, Cultura y Deporte.

Cabañas, M.D. (2009). Compendio de cineantropometría. Madrid: CTO Medicina.

Carter, J.E. (1975). The Heath-Carter somatotype method. San Diego: San Diego State University.

Carter, J.E., \& Ackland, T. (1994). Kinanthropometry in aquatic sports: a study of world class athletes (Vol. 5). Champaign (IL): HK Sport Science Monograph.

Chollet, D. (2003). Natación deportiva. Zaragoza: Inde Publicaciones.

Esparza, F. (1993). Manual de Cineantropometría. Pamplona: Grupo Español de Cineantropometría.

Fell, J., \& Gaffney, P. (2001). Physiological profiles of Australian surf boat rowers. Journal of Science and Medicine in Sport, 4(2), 188-195.

Ferragut, C., Vila, H., \& Rodríguez, N. (2007). Aspectos fisiológicos tener en cuenta en salvamento acuático deportivo. En JA. Abraldes y N. Rodríguez (Eds), El salvamento acuático deportivo a estudio. pp 163-181. Coruña: Federación de Salvamento e Socorrismo de Galicia.
González-Badillo, J.J., \& Gorostiaga, E. (2002). Fundamentos del entrenamiento de fuerza. Zaragoza: Inde Publicaciones.

Gorski, T., Rosser, T., Hoppeler, H., \& Vogt M. (2014). An anthropometric and physical profile of young swiss alpine skiers between 2004 and 2011. International journal of sports physiology and performance, 9(1), 108-16.

Gulbin, J., Fell, J., \& Gaffney, P. (1996). A physiological profile of elite surf ironmen, full time lifeguards and patrolling surf life savers. Australian Journal of Science and Medicine in Sports, 28(3), 86-90.

Heath, B.H., \& Carter, JE. (1980). A modified somatotype method. American Journal of Physical Anthropology, 27, 57-74.

Jürimäe, J., Haljaste, K., Cicchella, A., Lätt, E., Purge, P., Leppik, A., \& Jürimäe, T. (2007). Analysis of swimming performance from physical, physiological and biomechanical parameters in young swimmers. Pediatric Exercise Science, 19, 70-81.

Knechtle, B., Knechtle, P., \& Rosemann, T. (2011). Upper body skinfold thickness is related to race performance in male Ironman triathletes. International Journal of Sports Medicine, 32(1), 20-27.

Lee, R.C., Wang, Z, Heo, M., Ross, R, Janssen, I., \& Heymsfield, S.B. (2000). Total-body skeletal muscle mass: development and crossvalidation of anthropometric prediction models. American Journal of Clinical Nutrition, 72, 796-803.

Leone, M., Lariviere, G., \& Comtois, A.S. (2002). Discriminant analysis of anthropometric and biomotor variables among elite adolescent female athletes in four sports. Journal of Sports Sciences, 20(6), 443-449.

Liiv, H., Wyon, M.A., Jürimäe, T., Saar, M., Mäestu, J., \& Jürimäe J. (2013) Anthropometry, Somatotypes, and Aerobic Power in Ballet, Contemporary Dance, and DanceSport. Medical Problems of Performing Artists, 28(4), 207-11.

Malina, R.M., Bouchard, C., \& Bard-Or, O. (2004). Growth, maduration and physical activity. Illinois: Human Kinetics

Martínez, S., Pasquarelli, B.N., Romaguera, D., Arasa, C., Tauler, P., \& Aguiló, A. (2011). Anthropometric characteristics and nutritional profile of young amateur swimmers. Journal of Strength \& Conditioning Research, 25(4), 1126-1133.

Morais, J.E., Jesus, S., Lopes, V., Garrido, N., Silva, A., Marinho, D., \& Barbosa, T.M. (2012). Linking selected kinematic, anthropometric and hydrodynamic variables to young swimmer performance. Pediatric Exercise Science, 24(4), 649-664.

Norton, K., \& Olds, T. (2001). Morphological evolution of athletes over the 20th century: causes and consequences. Sports Medicine, 31(11), 763-783.

Rodríguez, N., Abraldes, J.A., \& Quintela, S. (2007). El salvamento deportivo en Galicia. Una década para la historia. En J.A. Abraldes y N. Rodríguez (Eds.), El salvamento acuático deportivo a estudio. pp. 23-59. La Coruña: Federación de Salvamento e Socorrismo de Galicia.

Rossi, M., Lyttle, A., El-Sallam, A., Benjanuvatra, N., \& Blanksby, B. (2013) Body Segment Inertial Parameters of elite swimmers Using DXA and indirect Methods. Journal of Sports Science and Medicine, 12(4), 761-75.

Scanlan, A., \& Dascombe, B. (2011). The anthropometric and performance characteristics of high-performance junior life savers. Serbian Journal of Sports Sciences, 5(2), 61-66.

Silva, A., Figueiredo, P., Abraldes, J.A., Marques, E.A., Soares, S., \& Fernandes, R.J. (2013). Sincronización entre miembros superiores en nadadores infantiles en la técnica de crol. Journal of Sport and Health Research, 5(3), 295-304.

Sinclair, W., Kerr, R., Spinks, W., \& Leicht, A. (2009). Blood lactate, heart rate and rating of perceived exertion responses of elite surf lifesavers to high-performance competition. Journal of Science and Medicine in Sport, 12(1), 101-106.

Stewart, A., Marfell-Jones, M., Olds, T., \& Ridden, H. (2011). International Standards for anthropometric assessment. New Zealand: Lower Hutt.

Strzala, M., \& Tyka, A. (2009). Physical endurance, somatic indices and swimming technique parameters as determinants of front crawl swimming speed at short distances in young swimmers. Medicina Sportiva, 13, 99-107.

Torras, R., Prats, T., Rodas, G., Riera, J., Viejo, A., \& Alfaro, V. (1995). Perfil fisiológico de los deportistas de salvamento acuático. Apunts. Medicina de l'Esport, 32(125), 157-163.

Tsekouras, Y.E., Kavouras, S.A., Campagna, A., Kotsis, Y.P., Syntosi, S.S., Papazoglou, K., \& Sidossis, L.S. (2005). The anthropometrical and physiological characteristics of elite water polo players. European Journal of Applied Physiology, 95(1), 35-41.

Vila, H., Manchado, C., Rodríguez, N., Abraldes, J.A., Alcaraz, P.E., \& Ferragut, C. (2012). Anthropometric profile, vertical jump, and throwing velocity in elite female handball players by playing positions. Journal of Strength \& Conditioning Research, 6(8), 2146-55.

Vila, H., Rodríguez, N., Ferragut, C., \& Abraldes, J.A. (2008). Perfil antropométrico de la selección gallega femenina de salvamento deportivo. FEGUI. Revista de salvamento acuático y primeros auxilios, 3(29), 57-60. 